\title{
Strong convergence theorem for total quasi- $\phi$-asymptotically nonexpansive mappings in a Banach space
}

\section{Siwaporn Saewan}

\section{"Correspondence:}

si_wa_pon@hotmail.com;

siwaporn@tsu.ac.th

Department of Mathematics and

Statistics, Faculty of Science, Thaksin

University (TSU), Paprayom,

Phatthalung 93110, Thailand

\begin{abstract}
In this paper, we prove strong convergence theorems to a point which is a fixed point of multi-valued mappings, a zero of an $\alpha$-inverse-strongly monotone operator and a solution of the equilibrium problem. Next, we obtain strong convergence theorems to a solution of the variational inequality problem, a fixed point of multi-valued mappings and a solution of the equilibrium problem. The results presented in this paper are improvement and generalization of the previously known results.
\end{abstract}

Keywords: total quasi- $\phi$-asymptotically nonexpansive multi-valued mappings; hybrid scheme; equilibrium problem; variational inequality problems; inverse-strongly monotone operator

\section{Introduction}

Let $E$ be a real Banach space with dual $E^{*}$, and let $C$ be a nonempty closed convex subset of $E$. Let $A: C \rightarrow E^{*}$ be an operator. $A$ is called monotone if

$$
\langle A x-A y, x-y\rangle \geq 0, \quad \forall x, y \in C
$$

$\alpha$-inverse-strongly monotone if there exists a constant $\alpha>0$ such that

$$
\langle A x-A y, x-y\rangle \geq \alpha\|A x-A y\|^{2}, \quad \forall x, y \in C ;
$$

L-Lipschitz continuous if there exists a constant $L>0$ such that

$$
\|A x-A y\| \leq L\|x-y\|, \quad \forall x, y \in C .
$$

If $A$ is $\alpha$-inverse strongly monotone, then it is $\frac{1}{\alpha}$-Lipschitz continuous, i.e.,

$$
\|A x-A y\| \leq \frac{1}{\alpha}\|x-y\|, \quad \forall x, y \in C .
$$

A monotone operator $A$ is said to be maximal if its graph $G(A)=\left\{\left(x, x^{*}\right): x^{*} \in A x\right\}$ is not properly contained in the graph of any other monotone operator.

Let $A$ be a monotone operator. We consider the problem of finding $x \in E$ such that

$$
0 \in A x,
$$

\section{Springer}

○2013 Saewan; licensee Springer. This is an Open Access article distributed under the terms of the Creative Commons Attribution License (http://creativecommons.org/licenses/by/2.0), which permits unrestricted use, distribution, and reproduction in any medium, provided the original work is properly cited. 
a point $x \in E$ is called a zero point of $A$. Denote by $A^{-1} 0$ the set of all points $x \in E$ such that $0 \in A x$. This problem is very important in optimization theory and related fields.

Let $A$ be a monotone operator. The classical variational inequality problem for an operator $A$ is to find $\hat{z} \in C$ such that

$$
\langle A \hat{z}, y-\hat{z}\rangle \geq 0, \quad \forall y \in C .
$$

The set of solutions of (1.2) is denoted by $V I(A, C)$. This problem is connected with the convex minimization problem, the complementary problem, the problem of finding a point $x \in E$ satisfying $A x=0$.

The value of $x^{*} \in E^{*}$ at $x \in E$ will be denoted by $\left\langle x, x^{*}\right\rangle$ or $x^{*}(x)$. For each $p>1$, the generalized duality mapping $J_{p}: E \rightarrow 2^{E^{*}}$ is defined by

$$
J_{p}(x)=\left\{x^{*} \in E^{*}:\left\langle x, x^{*}\right\rangle=\|x\|^{p},\left\|x^{*}\right\|=\|x\|^{p-1}\right\}
$$

for all $x \in E$. In particular, $J=J_{2}$ is called the normalized duality mapping. If $E$ is a Hilbert space, then $J=I$, where $I$ is the identity mapping.

Consider the functional defined by

$$
\phi(y, x)=\|y\|^{2}-2\langle y, J x\rangle+\|x\|^{2} \quad \text { for } x, y \in E,
$$

where $J$ is the normalized duality mapping. It is obvious from the definition of $\phi$ that

$$
(\|y\|-\|x\|)^{2} \leq \phi(y, x) \leq(\|y\|+\|x\|)^{2}, \quad \forall x, y \in E .
$$

Alber [1] introduced that the generalized projection $\Pi_{C}: E \rightarrow C$ is a map that assigns to an arbitrary point $x \in E$ the minimum point of the functional $\phi(x, y)$, that is, $\Pi_{C} x=\bar{x}$, where $\bar{x}$ is the solution of the minimization problem

$$
\phi(\bar{x}, x)=\inf _{y \in C} \phi(y, x)
$$

existence and uniqueness of the operator $\Pi_{C}$ follows from the properties of the functional $\phi(x, y)$ and strict monotonicity of the mapping $J$.

Iiduka and Takahashi [2] introduced the following iterative scheme for finding a solution of the variational inequality problem for an inverse-strongly monotone operator $A$ in a 2-uniformly convex and uniformly smooth Banach space $E: x_{1}=x \in C$ and

$$
x_{n+1}=\Pi_{C} J^{-1}\left(J x_{n}-\lambda_{n} A x_{n}\right), \quad \forall n \geq 1,
$$

where $\Pi_{C}$ is the generalized projection from $E$ onto $C, J$ is the duality mapping from $E$ into $E^{*}$ and $\left\{\lambda_{n}\right\}$ is a sequence of positive real numbers. They proved that the sequence $\left\{x_{n}\right\}$ generated by (1.6) converges weakly to some element of $V I(A, C)$. In connection, Iiduka and Takahashi [3] studied the following iterative scheme for finding a zero point of a monotone 
operator $A$ in a 2-uniformly convex and uniformly smooth Banach space $E$ :

$$
\left\{\begin{array}{l}
x_{1}=x \in E \quad \text { chosen arbitrarily, } \\
y_{n}=J^{-1}\left(J x_{n}-\lambda_{n} A x_{n}\right) \\
X_{n}=\left\{z \in E: \phi\left(z, y_{n}\right) \leq \phi\left(z, x_{n}\right)\right\} \\
Y_{n+1}=\left\{z \in E:\left\langle x_{n}-z, J x-J x_{n}\right\rangle \geq 0\right\} \\
x_{n+1}=\Pi_{X_{n} \cap Y_{n}}(x),
\end{array}\right.
$$

where $\Pi_{X_{n} \cap Y_{n}}$ is the generalized projection from $E$ onto $X_{n} \cap Y_{n}, J$ is the duality mapping from $E$ into $E^{*}$ and $\left\{\lambda_{n}\right\}$ is a sequence of positive real numbers. They proved that the sequence $\left\{x_{n}\right\}$ converges strongly to an element of $A^{-1} 0$. Moreover, under the additional suitable assumption they proved that the sequence $\left\{x_{n}\right\}$ converges strongly to some element of $V I(A, C)$. Some solution methods have been proposed to solve the variational inequality problem; see, for instance, [4-6].

A mapping $T: C \rightarrow C$ is said to be $\phi$-nonexpansive $[7,8]$ if

$$
\phi(T x, T y) \leq \phi(x, y), \quad \forall x, y \in C .
$$

$T$ is said to be quasi- $\phi$-nonexpansive $[7,8]$ if $F(T) \neq \emptyset$ and

$$
\phi(p, T x) \leq \phi(p, x), \quad \forall x \in C, p \in F(T) .
$$

$T$ is said to be total quasi- $\phi$-asymptotically nonexpansive, if $F(T) \neq \emptyset$ and there exist nonnegative real sequences $v_{n}, \mu_{n}$ with $v_{n} \rightarrow 0, \mu_{n} \rightarrow 0$ as $n \rightarrow \infty$ and a strictly increasing continuous function $\varphi: \mathbb{R}^{+} \rightarrow \mathbb{R}^{+}$with $\varphi(0)=0$ such that

$$
\phi\left(p, T^{n} x\right) \leq \phi(p, x)+v_{n} \varphi(\phi(p, x))+\mu_{n}, \quad \forall n \geq 1, \forall x \in C, p \in F(T) .
$$

Let $2^{C}$ be the family of all nonempty subsets of $C$, and let $S: C \rightarrow 2^{C}$ be a multi-valued mapping. For a point $q \in C, n \geq 1$ define an iterative sequence as follows:

$$
\begin{aligned}
& S q:=\left\{q_{1}: q_{1} \in S q\right\}, \\
& S^{2} q=S S q:=\bigcup_{q_{1} \in S q} S q_{1}, \\
& S^{3} q=S S^{2} q:=\bigcup_{q_{2} \in T^{2} q} S q_{2}, \\
& \vdots \\
& S^{n} q=S S^{n-1} q:=\bigcup_{q_{n-1} \in S^{n-1} q} S q_{n-1} .
\end{aligned}
$$

A point $p \in C$ is said to be an asymptotic fixed point of $S$ if there exists a sequence $\left\{x_{n}\right\}$ in $C$ such that $\left\{x_{n}\right\}$ converges weakly to $p$ and

$$
\lim _{n \rightarrow \infty} d\left(x_{n}, S x_{n}\right):=\lim _{n \rightarrow \infty} \inf _{x \in S x_{n}}\left\|x_{n}-x\right\|=0 .
$$

The asymptotic fixed point set of $S$ is denoted by $\widehat{F}(S)$. 
A multi-valued mapping $S$ is said to be total quasi- $\phi$-asymptotically nonexpansive if $F(S) \neq \emptyset$ and there exist nonnegative real sequences $v_{n}, \mu_{n}$ with $v_{n} \rightarrow 0, \mu_{n} \rightarrow 0$ as $n \rightarrow \infty$ and a strictly increasing continuous function $\varphi: \mathbb{R}^{+} \rightarrow \mathbb{R}^{+}$with $\varphi(0)=0$ such that for all $x \in C, p \in F(S)$,

$$
\phi\left(p, w_{n}\right) \leq \phi(p, x)+v_{n} \varphi(\phi(p, x))+\mu_{n}, \quad \forall n \geq 1, w_{n} \in S^{n} x .
$$

$S$ is said to be closed if for any sequence $\left\{x_{n}\right\}$ and $\left\{w_{n}\right\}$ in $C$ with $w_{n} \in S x_{n}$ if $x_{n} \rightarrow x$ and $w_{n} \rightarrow w$, then $w \in S x$.

A multi-valued mapping $S$ is said to be uniformly asymptotically regular on $C$ if

$$
\lim _{n \rightarrow \infty}\left(\sup _{x \in C}\left\|s_{n+1}-s_{n}\right\|\right)=0, \quad s_{n} \in S^{n} x .
$$

Every quasi- $\phi$-asymptotically nonexpansive multi-valued mapping implies a quasi- $\phi$ asymptotically nonexpansive mapping but the converse is not true.

In 2012, Chang et al. [9] introduced the concept of total quasi- $\phi$-asymptotically nonexpansive multi-valued mapping and then proved some strong convergence theorem by using the hybrid shrinking projection method.

Let $f: C \times C \rightarrow \mathbb{R}$ be a bifunction, the equilibrium problem is to find $x \in C$ such that

$$
f(x, y) \geq 0, \quad \forall y \in C
$$

The set of solutions of (1.8) is denoted by $E P(f)$. The equilibrium problem is very general in the sense that it includes, as special cases, optimization problems, variational inequality problems, min-max problems, saddle point problem, fixed point problem, Nash EP. In 2008, Takahashi and Zembayashi $[10,11]$ introduced iterative sequences for finding a common solution of an equilibrium problem and a fixed point problem. Some solution methods have been proposed to solve the equilibrium problem; see, for instance, [12-21].

For a mapping $A: C \rightarrow E^{*}$, let $f(x, y)=\langle A x, y-x\rangle$ for all $x, y \in C$. Then $x \in E P(f)$ if and only if $\langle T x, y-x\rangle \geq 0$ for all $y \in C$; i.e., $x$ is a solution of the variational inequality.

Motivated and inspired by the work mentioned above, in this paper, we introduce and prove strong convergence of a new hybrid projection algorithm for a fixed point of total quasi- $\phi$-asymptotically nonexpansive multi-valued mappings, the solution of the equilibrium problem, a zero point of monotone operators. Moreover, we prove strong convergence to the solution of the variation inequality in a uniformly smooth and 2-uniformly convex Banach space.

\section{Preliminaries}

A Banach space $E$ with the norm $\|\cdot\|$ is called strictly convex if $\left\|\frac{x+y}{2}\right\|<1$ for all $x, y \in E$ with $\|x\|=\|y\|=1$ and $x \neq y$. Let $U=\{x \in E:\|x\|=1\}$ be the unit sphere of $E$. A Banach space $E$ is called smooth if the $\operatorname{limit}_{\lim } \lim _{t \rightarrow 0} \frac{\|x+t y\|-\|x\|}{t}$ exists for each $x, y \in U$. It is also called uniformly smooth if the limit exists uniformly for all $x, y \in U$. The modulus of convexity of $E$ is the function $\delta:[0,2] \rightarrow[0,1]$ defined by

$$
\delta(\varepsilon)=\inf \left\{1-\left\|\frac{x+y}{2}\right\|: x, y \in E,\|x\|=\|y\|=1,\|x-y\| \geq \varepsilon\right\} .
$$


A Banach space $E$ is uniformly convex if and only if $\delta(\varepsilon)>0$ for all $\varepsilon \in(0,2]$. Let $p$ be a fixed real number with $p \geq 2$. A Banach space $E$ is said to be $p$-uniformly convex if there exists a constant $c>0$ such that $\delta(\varepsilon) \geq c \varepsilon^{p}$ for all $\varepsilon \in[0,2]$. Observe that every $p$-uniform convex is uniformly convex. Every uniformly convex Banach space $E$ has the Kadec-Klee property, that is, for any sequence $\left\{x_{n}\right\} \subset E$, if $x_{n} \rightarrow x \in E$ and $\left\|x_{n}\right\| \rightarrow\|x\|$, then $x_{n} \rightarrow x$.

Let $E$ be a real Banach space with dual $E^{*}, E$ is uniformly smooth if and only if $E^{*}$ is a uniformly convex Banach space. If $E$ is a uniformly smooth Banach space, then $E$ is a smooth and reflexive Banach space.

\section{Remark 2.1}

- If $E$ is uniformly smooth, then $J$ is uniformly norm-to-norm continuous on each bounded subset of $E$.

- If $E$ is reflexive smooth and strictly convex, then the normalized duality mapping $J$ is single-valued, one-to-one and onto.

- If $E$ is a reflexive strictly convex and smooth Banach space and $J$ is the duality mapping from $E$ into $E^{*}$, then $J^{-1}$ is also single-valued, bijective and is also the duality mapping from $E^{*}$ into $E$ and thus $J J^{-1}=I_{E^{*}}$ and $J^{-1} J=I_{E}$.

See [22] for more details.

Remark 2.2 If $E$ is a reflexive, strictly convex and smooth Banach space, then $\phi(x, y)=0$ if and only if $x=y$. It is sufficient to show that if $\phi(x, y)=0$, then $x=y$. From (1.3) we have $\|x\|=\|y\|$. This implies that $\langle x, J y\rangle=\|x\|^{2}=\|J y\|^{2}$. From the definition of $J$, one has $J x=J y$. Therefore, we have $x=y$ (see [22, 23] for more details).

Lemma 2.3 (Beauzamy [24] and Xu [25]) If E is a 2-uniformly convex Banach space, then, for all $x, y \in E$, we have

$$
\|x-y\| \leq \frac{2}{c^{2}}\|x-J y\|
$$

where $J$ is the normalized duality mapping of $E$ and $0<c \leq 1$.

The best constant $\frac{1}{c}$ in the lemma is called the $p$-uniformly convex constant of $E$.

Lemma 2.4 (Beauzamy [24] and Zalinescu [26]) If $E$ is a p-uniformly convex Banach space, and let $p$ be a given real number with $p \geq 2$, then, for all $x, y \in E, J_{x} \in J_{p}(x)$ and $J_{y} \in J_{p}(y)$,

$$
\left\langle x-y, J_{x}-J_{y}\right\rangle \geq \frac{c^{p}}{2^{p-2} p}\|x-y\|^{p},
$$

where $J_{p}$ is the generalized duality mapping of $E$ and $\frac{1}{c}$ is the p-uniformly convex constant of $E$.

Lemma 2.5 (Kamimura and Takahashi [27]) Let E be a uniformly convex and smooth Banach space, and let $\left\{x_{n}\right\},\left\{y_{n}\right\}$ be two sequences of $E$. If $\phi\left(x_{n}, y_{n}\right) \rightarrow 0$ and either $\left\{x_{n}\right\}$ or $\left\{y_{n}\right\}$ is bounded, then $\left\|x_{n}-y_{n}\right\| \rightarrow 0$. 
Lemma 2.6 (Alber [1]) Let $C$ be a nonempty closed convex subset of a smooth Banach space $E$, and let $x \in E$. Then $x_{0}=\Pi_{C} x$ if and only if

$$
\left\langle x_{0}-y, J x-J x_{0}\right\rangle \geq 0, \quad \forall y \in C .
$$

Lemma 2.7 (Alber [1]) Let E be a reflexive strictly convex and smooth Banach space, $C$ be a nonempty closed convex subset of $E$, and let $x \in E$. Then

$$
\phi\left(y, \Pi_{C} x\right)+\phi\left(\Pi_{C} x, x\right) \leq \phi(y, x), \quad \forall y \in C .
$$

Lemma 2.8 (Chang et al. [9]) Let C be a nonempty, closed and convex subset of a uniformly smooth and strictly convex Banach space $E$ with the Kadec-Klee property. Let $S: C \rightarrow 2^{C}$ be a closed and total quasi- $\phi$-asymptotically nonexpansive multi-valued mapping with nonnegative real sequence $v_{n}$ and $\mu_{n}$ with $v_{n} \rightarrow 0, \mu_{n} \rightarrow 0$ as $n \rightarrow \infty$ and a strictly increasing continuous function $\varphi: \mathbb{R}^{+} \rightarrow \mathbb{R}^{+}$with $\varphi(0)=0$. If $\mu_{1}=0$, then the fixed point set $F(S)$ is a closed convex subset of $C$.

For solving the equilibrium problem for a bifunction $f: C \times C \rightarrow \mathbb{R}$, let us assume that $f$ satisfies the following conditions:

(A1) $f(x, x)=0$ for all $x \in C$;

(A2) $f$ is monotone, i.e., $f(x, y)+f(y, x) \leq 0$ for all $x, y \in C$;

(A3) for each $x, y, z \in C$,

$$
\lim _{t \downarrow 0} f(t z+(1-t) x, y) \leq f(x, y)
$$

(A4) for each $x \in C, y \mapsto f(x, y)$ is convex and lower semi-continuous.

Lemma 2.9 (Blum and Oettli [28]) Let $C$ be a closed convex subset of a smooth, strictly convex and reflexive Banach space $E$, let $f$ be a bifunction from $C \times C$ to $\mathbb{R}$ satisfying (A1)(A4), and let $r>0$ and $x \in E$. Then there exists $z \in C$ such that

$$
f(z, y)+\frac{1}{r}\langle y-z, J z-J x\rangle \geq 0, \quad \forall y \in C .
$$

Lemma 2.10 (Takahashi and Zembayashi [11]) Let C be a closed convex subset of a uniformly smooth, strictly convex and reflexive Banach space $E$, and let $f$ be a bifunction from $C \times C$ to $\mathbb{R}$ satisfying conditions (A1)-(A4). For all $r>0$ and $x \in E$, define a mapping $T_{r}: E \rightarrow C$ as follows:

$$
T_{r} x=\left\{z \in C: f(z, y)+\frac{1}{r}\langle y-z, J z-J x\rangle \geq 0, \forall y \in C\right\} .
$$

Then the following hold:

(1) $T_{r}$ is single-valued;

(2) $T_{r}$ is a firmly nonexpansive-type mapping [29], that is, for all $x, y \in E$,

$$
\left\langle T_{r} x-T_{r} y, J T_{r} x-J T_{r} y\right\rangle \leq\left\langle T_{r} x-T_{r} y, J x-J y\right\rangle ;
$$


(3) $F\left(T_{r}\right)=E P(f)$;

(4) $E P(f)$ is closed and convex.

Lemma 2.11 (Takahashi and Zembayashi [11]) Let $C$ be a closed convex subset of a smooth, strictly convex and reflexive Banach space $E$, let $f$ be a bifunction from $C \times C$ to $\mathbb{R}$ satisfying (A1)-(A4), and let $r>0$. Then, for $x \in E$ and $q \in F\left(T_{r}\right)$,

$$
\phi\left(q, T_{r} x\right)+\phi\left(T_{r} x, x\right) \leq \phi(q, x)
$$

Let $A$ be an inverse-strongly monotone mapping of $C$ into $E^{*}$ which is said to be hemicontinuous if for all $x, y \in C$, the mapping $h$ of $[0,1]$ into $E^{*}$, defined by $h(t)=A(t x+(1-t) y)$, is continuous with respect to the weak* topology of $E^{*}$. We define by $N_{C}(v)$ the normal cone for $C$ at a point $v \in C$, that is,

$$
N_{C}(v)=\left\{x^{*} \in E^{*}:\left\langle v-y, x^{*}\right\rangle \geq 0, \forall y \in C\right\}
$$

Theorem 2.12 (Rockafellar [30]) Let C be a nonempty, closed convex subset of a Banach space $E$, and let $A$ be a monotone, hemicontinuous operator of $C$ into $E^{*}$. Let $B \subset E \times E^{*}$ be an operator defined as follows:

$$
B v= \begin{cases}A v+N_{C}(v), & v \in C ; \\ \emptyset, & \text { otherwise. }\end{cases}
$$

Then $B$ is maximal monotone and $B^{-1} 0=V I(A, C)$.

Theorem 2.13 (Takahashi [31]) Let $C$ be a nonempty subset of a Banach space E, and let $A$ be a monotone, hemicontinuous operator of $C$ into $E^{*}$ with $C=D(A)$. Then

$$
V I(A, C)=\{u \in C:\langle v-u, A v\rangle \geq 0, \forall v \in C\}
$$

It is obvious that the set $\operatorname{VI}(A, C)$ is a closed and convex subset of $C$ and the set $A^{-1} 0=$ $V I(A, E)$ is a closed and convex subset of $E$.

Theorem 2.14 (Takahashi [31]) Let $C$ be a nonempty compact convex subset of a Banach space $E$, and let $A$ be a monotone, hemicontinuous operator of $C$ into $E^{*}$ with $C=D(A)$. Then $\operatorname{VI}(A, C)$ is nonempty.

We make use of the following mapping $V$ studied in Alber [1]:

$$
V\left(x, x^{*}\right)=\|x\|^{2}-2\left\langle x, x^{*}\right\rangle+\left\|x^{*}\right\|^{2}, \quad \forall x \in E, x^{*} \in E^{*},
$$

that is, $V\left(x, x^{*}\right)=\phi\left(x, J^{-1}\left(x^{*}\right)\right)$.

Lemma 2.15 (Alber [1]) Let E be a reflexive strictly convex smooth Banach space, and let $V$ be as in (2.4). Then we have

$$
V\left(x, x^{*}\right)+2\left\langle J^{-1}\left(x^{*}\right)-x, y^{*}\right\rangle \leq V\left(x, x^{*}+y^{*}\right), \quad \forall x \in E, x^{*}, y^{*} \in E^{*} .
$$


Lemma 2.16 (Beauzamy [24] and Xu [25]) If $E$ is a 2-uniformly convex Banach space, then, for all $x, y \in E$, we have

$$
\|x-y\| \leq \frac{2}{c^{2}}\|J x-J y\|
$$

where $J$ is the normalized duality mapping of $E$ and $0<c \leq 1$.

Lemma 2.17 (Cho et al. [32]) Let $E$ be a uniformly convex Banach space, and let $B_{r}(0)=$ $\{x \in E:\|x\| \leq r\}$ be a closed ball of $E$. Then there exists a continuous strictly increasing convex function $g:[0, \infty) \rightarrow[0, \infty)$ with $g(0)=0$ such that

$$
\|\lambda x+\mu y+\gamma z\|^{2} \leq\|\lambda x\|^{2}+\|\mu y\|^{2}+\|\gamma z\|^{2}-\lambda \mu g(\|x-y\|)
$$

for all $x, y, z \in B_{r}(0)$ and $\lambda, \mu, \gamma \in[0,1]$ with $\lambda+\mu+\gamma=1$.

Lemma 2.18 (Pascali and Sburlan [33]) Let E be a real smooth Banach space, and let A : $E \rightarrow 2^{E^{*}}$ be a maximal monotone mapping. Then $A^{-1} 0$ is a closed and convex subset of $E$ and the graph $G(A)$ of $A$ is demiclosed in the following sense: if $\left\{x_{n}\right\} \subset D(A)$ with $x_{n} \rightarrow x \in E$ and $y_{n} \in A x_{n}$ with $y_{n} \rightarrow y \in E^{*}$, then $x \in D(A)$ and $y \in A x$.

\section{Main results}

Theorem 3.1 Let $C$ be a nonempty closed and convex subset of a uniformly smooth and 2-uniformly convex Banach space E. Letf be a bifunction from $C \times C$ to $\mathbb{R}$ satisfying conditions (A1)-(A4), and let $A$ be an $\alpha$-inverse-strongly monotone mapping of $E$ into $E^{*}$. Let $S: C \rightarrow 2^{C}$ be a closed and total quasi- $\phi$-asymptotically nonexpansive multi-valued mapping with nonnegative real sequences $v_{n}, \mu_{n}$ with $v_{n} \rightarrow 0, \mu_{n} \rightarrow 0$ as $n \rightarrow \infty$ and a strictly increasing continuous function $\psi: \mathbb{R}^{+} \rightarrow \mathbb{R}^{+}$with $\psi(0)=0$. Assume that $S$ is uniformly asymptotically regular on $C$ with $\mu_{1}=0$ and $F:=F(S) \cap E P(f) \cap A^{-1} 0 \neq \emptyset$. For arbitrary $x_{1} \in C, C_{1}=C$, generate a sequence $\left\{x_{n}\right\}$ by

$$
\left\{\begin{array}{l}
z_{n}=J^{-1}\left(J x_{n}-\lambda_{n} A x_{n}\right), \\
u_{n}=T_{r_{n}} z_{n}, \\
y_{n}=J^{-1}\left(\alpha_{n} J x_{n}+\beta_{n} J w_{n}+\gamma_{n} J u_{n}\right), \quad w_{n} \in S^{n} x_{n}, \\
C_{n+1}=\left\{v \in C_{n}: \phi\left(v, y_{n}\right) \leq \phi\left(v, z_{n}\right) \leq \phi\left(v, x_{n}\right)+K_{n}\right\} \\
x_{n+1}=\Pi_{C_{n+1}} x_{1}, \quad n \in \mathbb{N},
\end{array}\right.
$$

where $K_{n}=v_{n} \sup _{q \in F} \psi\left(\phi\left(q, x_{n}\right)\right)+\mu_{n}$. Assume that the control sequences $\left\{\alpha_{n}\right\},\left\{\beta_{n}\right\},\left\{\gamma_{n}\right\}$, $\left\{\lambda_{n}\right\}$ and $\left\{r_{n}\right\}$ satisfy the following conditions:

1. $\left\{\alpha_{n}\right\},\left\{\beta_{n}\right\}$ and $\left\{\gamma_{n}\right\}$ are sequences in $(0,1)$ such that $\alpha_{n}+\beta_{n}+\gamma_{n}=1$, $\liminf _{n \rightarrow \infty} \alpha_{n} \beta_{n}>0$,

2. $\left\{\lambda_{n}\right\} \subset[a, b]$ for some $a, b$ with $0<a<b<\frac{c^{2} \alpha}{2}$ and $\frac{1}{c}$ is the 2-uniformly convex constant of $E$,

3. $\left\{r_{n}\right\} \subset[d, \infty)$ for some $d>0$,

then $\left\{x_{n}\right\}$ converges strongly to $\Pi_{F} x_{1}$. 
Proof We will show that $C_{n}$ is closed and convex for all $n \in \mathbb{N}$. Since $C_{1}=C$ is closed and convex. Suppose that $C_{n}$ is closed and convex for all $n \in \mathbb{N}$. For any $v \in C_{n}$, we know that $\phi\left(v, y_{n}\right) \leq \phi\left(v, x_{n}\right)+K_{n}$ is equivalent to

$$
2\left\langle v, J x_{n}-J y_{n}\right\rangle \leq\left\|x_{n}\right\|^{2}-\left\|y_{n}\right\|^{2}+K_{n} .
$$

That is, $C_{n+1}$ is closed and convex, hence $C_{n}$ is closed and convex for all $n \in \mathbb{N}$.

We show by induction that $F \subset C_{n}$ for all $n \in \mathbb{N}$. It is obvious that $F \subset C=C_{1}$. Suppose that $F \subset C_{n}$ where $n \in \mathbb{N}$. Let $q \in F$, we have

$$
\begin{aligned}
\phi\left(q, z_{n}\right) & =\phi\left(q, J^{-1}\left(J x_{n}-\lambda_{n} A x_{n}\right)\right) \\
& =V\left(q, J x_{n}-\lambda_{n} A x_{n}\right) \\
& \leq V\left(q,\left(J x_{n}-\lambda_{n} A x_{n}\right)+\lambda_{n} A x_{n}\right)-2\left\langle J^{-1}\left(J x_{n}-\lambda_{n} A x_{n}\right)-q, \lambda_{n} A x_{n}\right\rangle \\
& =V\left(q, J x_{n}\right)-2 \lambda_{n}\left\langle J^{-1}\left(J x_{n}-\lambda_{n} A x_{n}\right)-q, A x_{n}\right\rangle \\
& =\phi\left(q, x_{n}\right)-2 \lambda_{n}\left\langle x_{n}-q, A x_{n}\right\rangle+2\left\langle J^{-1}\left(J x_{n}-\lambda_{n} A x_{n}\right)-x_{n},-\lambda_{n} A x_{n}\right\rangle .
\end{aligned}
$$

Since $A$ is an $\alpha$-inverse-strongly monotone mapping, we get

$$
\begin{aligned}
-2 \lambda_{n}\left\langle x_{n}-q, A x_{n}\right\rangle & =-2 \lambda_{n}\left\langle x_{n}-q, A x_{n}-A q\right\rangle-2 \lambda_{n}\left\langle x_{n}-q, A q\right\rangle \\
& \leq-2 \lambda_{n}\left\langle x_{n}-q, A x_{n}-A q\right\rangle \\
& =-2 \alpha \lambda_{n}\left\|A x_{n}-A q\right\|^{2} .
\end{aligned}
$$

It follows from Lemma 2.17 that

$$
\begin{aligned}
2\left\langle J^{-1}\left(J x_{n}-\lambda_{n} A x_{n}\right)-x_{n},-\lambda_{n} A x_{n}\right\rangle & =2\left\langle J^{-1}\left(J x_{n}-\lambda_{n} A x_{n}\right)-J^{-1}\left(J x_{n}\right),-\lambda_{n} A x_{n}\right\rangle \\
& \leq 2\left\|J^{-1}\left(J x_{n}-\lambda_{n} A x_{n}\right)-J^{-1}\left(J x_{n}\right)\right\|\left\|\lambda_{n} A x_{n}\right\| \\
& \leq \frac{4}{c^{2}}\left\|J J^{-1}\left(J x_{n}-\lambda_{n} A x_{n}\right)-J J^{-1}\left(J x_{n}\right)\right\|\left\|\lambda_{n} A x_{n}\right\| \\
& =\frac{4}{c^{2}}\left\|x_{n}-\lambda_{n} A x_{n}-J x_{n}\right\|\left\|\lambda_{n} A x_{n}\right\| \\
& =\frac{4}{c^{2}}\left\|\lambda_{n} A x_{n}\right\|^{2} \\
& =\frac{4}{c^{2}} \lambda_{n}^{2}\left\|A x_{n}\right\|^{2} \\
& \leq \frac{4}{c^{2}} \lambda_{n}^{2}\left\|A x_{n}-A q\right\|^{2} .
\end{aligned}
$$

Replacing (3.2) by (3.3) and (3.4), we get

$$
\begin{aligned}
\phi\left(q, z_{n}\right) & \leq \phi\left(q, x_{n}\right)-2 \alpha \lambda_{n}\left\|A x_{n}-A q\right\|^{2}+\frac{4}{c^{2}} \lambda_{n}^{2}\left\|A x_{n}-A q\right\|^{2} \\
& =\phi\left(q, x_{n}\right)+2 \lambda_{n}\left(\frac{2}{c^{2}} \lambda_{n}-\alpha\right)\left\|A x_{n}-A q\right\|^{2} \\
& \leq \phi\left(q, x_{n}\right) .
\end{aligned}
$$


From Lemma 2.11, we know that

$$
\phi\left(q, u_{n}\right)=\phi\left(q, T_{r_{n}} z_{n}\right) \leq \phi\left(q, z_{n}\right) \leq \phi\left(q, x_{n}\right) .
$$

Since $S$ is a total quasi- $\phi$-asymptotically nonexpansive multi-valued mapping and $w_{n} \in$ $S^{n} x_{n}$, it follows that

$$
\begin{aligned}
\phi\left(q, y_{n}\right) & =\phi\left(q, J^{-1}\left(\alpha_{n} J x_{n}+\beta_{n} J w_{n}+\gamma_{n} J u_{n}\right)\right) \\
& =\|q\|^{2}-2\left\langle q, \alpha_{n} J x_{n}+\beta_{n} J w_{n}+\gamma_{n} J u_{n}\right\rangle+\left\|\alpha_{n} J x_{n}+\beta_{n} J w_{n}+\gamma_{n} J u_{n}\right\|^{2} \\
& \leq \alpha_{n} \phi\left(q, x_{n}\right)+\beta_{n} \phi\left(q, w_{n}\right)+\gamma_{n} \phi\left(q, u_{n}\right) \\
& \leq \alpha_{n} \phi\left(q, x_{n}\right)+\beta_{n} \phi\left(q, x_{n}\right)+\beta_{n} v_{n} \psi\left(\phi\left(q, x_{n}\right)\right)+\beta_{n} \mu_{n}+\gamma_{n} \phi\left(q, u_{n}\right) \\
& \leq \alpha_{n} \phi\left(q, x_{n}\right)+\beta_{n} \phi\left(q, x_{n}\right)+v_{n} \sup _{q \in F} \psi\left(\phi\left(q, x_{n}\right)\right)+\mu_{n}+\gamma_{n} \phi\left(q, u_{n}\right) \\
& =\alpha_{n} \phi\left(q, x_{n}\right)+\beta_{n} \phi\left(q, x_{n}\right)+\gamma_{n} \phi\left(q, u_{n}\right)+K_{n} \\
& \leq \alpha_{n} \phi\left(q, x_{n}\right)+\beta_{n} \phi\left(q, x_{n}\right)+\gamma_{n} \phi\left(q, T_{r_{n}} z_{n}\right)+K_{n} \\
& \leq \alpha_{n} \phi\left(q, x_{n}\right)+\beta_{n} \phi\left(q, x_{n}\right)+\gamma_{n} \phi\left(q, z_{n}\right)+K_{n} \\
& \leq \alpha_{n} \phi\left(q, x_{n}\right)+\beta_{n} \phi\left(q, x_{n}\right)+\gamma_{n} \phi\left(q, x_{n}\right)+K_{n} \\
& \leq \phi\left(q, x_{n}\right)+K_{n},
\end{aligned}
$$

where $K_{n}=v_{n} \sup _{q \in F} \psi\left(\phi\left(q, x_{n}\right)\right)+\mu_{n}$.

This shows that $q \in C_{n+1}$, which implies that $F \subset C_{n+1}$. Hence $F \subset C_{n}$ for all $n \in \mathbb{N}$ and the sequence $\left\{x_{n}\right\}$ is well defined.

From the definition of $C_{n+1}$ with $x_{n}=\Pi_{C_{n}} x_{1}$ and $x_{n+1}=\Pi_{C_{n+1}} x_{1} \in C_{n+1} \subset C_{n}$, it follows that

$$
\phi\left(x_{n}, x_{1}\right) \leq \phi\left(x_{n+1}, x_{1}\right), \quad \forall n \geq 1,
$$

that is, $\left\{\phi\left(x_{n}, x_{1}\right)\right\}$ is nondecreasing. By Lemma 2.7, we get

$$
\begin{aligned}
\phi\left(x_{n}, x_{1}\right) & =\phi\left(\Pi_{C_{n}} x_{1}, x_{1}\right) \\
& \leq \phi\left(q, x_{1}\right)-\phi\left(q, x_{n}\right) \\
& \leq \phi\left(q, x_{1}\right), \quad \forall q \in F .
\end{aligned}
$$

This implies that $\left\{\phi\left(x_{n}, x_{1}\right)\right\}$ is bounded and so $\lim _{n \rightarrow \infty} \phi\left(x_{n}, x_{1}\right)$ exists. In particular, by (1.4), the sequence $\left\{\left(\left\|x_{n}\right\|-\left\|x_{1}\right\|\right)^{2}\right\}$ is bounded. This implies $\left\{x_{n}\right\}$ is also bounded. So, we have $\left\{u_{n}\right\},\left\{z_{n}\right\}$ and $\left\{y_{n}\right\}$ are also bounded.

Since $x_{m}=\Pi_{C_{m}} x_{1} \in C_{m} \subset C_{n}$ for all $m, n \geq 1$ with $m>n$, by Lemma 2.7, we have

$$
\begin{aligned}
\phi\left(x_{m}, x_{n}\right) & =\phi\left(x_{m}, \Pi_{C_{n}} x_{1}\right) \\
& \leq \phi\left(x_{m}, x_{1}\right)-\phi\left(\Pi_{C_{n}} x_{1}, x_{1}\right) \\
& =\phi\left(x_{m}, x_{1}\right)-\phi\left(x_{n}, x_{1}\right),
\end{aligned}
$$


taking $m, n \rightarrow \infty$, we have $\phi\left(x_{m}, x_{n}\right) \rightarrow 0$. This implies that $\left\{x_{n}\right\}$ is a Cauchy sequence. From Lemma 2.5, it follows that $\left\|x_{n}-x_{m}\right\| \rightarrow 0$ and $\left\{x_{n}\right\}$ is a Cauchy sequence. By the completeness of $E$ and the closedness of $C$, we can assume that there exists $p \in C$ such that

$$
\lim _{n \rightarrow \infty} x_{n}=p
$$

we also get that

$$
\lim _{n \rightarrow \infty} K_{n}=\lim _{n \rightarrow \infty} v_{n} \sup _{q \in F} \psi\left(\phi\left(q, x_{n}\right)\right)+\mu_{n}=0 .
$$

Next, we show that $p \in F:=F(S) \cap A^{-1} 0 \cap E P(f)$.

(a) We show that $p \in F(S)$. By the definition of $\Pi_{C_{n}} x_{1}$, we have

$$
\begin{aligned}
\phi\left(x_{n+1}, x_{n}\right) & =\phi\left(x_{n+1}, \Pi_{C_{n}} x_{1}\right) \\
& \leq \phi\left(x_{n+1}, x_{1}\right)-\phi\left(\Pi_{C_{n}} x_{1}, x_{1}\right) \\
& =\phi\left(x_{n+1}, x_{1}\right)-\phi\left(x_{n}, x_{1}\right) .
\end{aligned}
$$

Since $\lim _{n \rightarrow \infty} \phi\left(x_{n}, x_{1}\right)$ exists, we get

$$
\lim _{n \rightarrow \infty} \phi\left(x_{n+1}, x_{n}\right)=0
$$

It follows from Lemma 2.5 that

$$
\lim _{n \rightarrow \infty}\left\|x_{n+1}-x_{n}\right\|=0
$$

From the definition of $C_{n+1}$ and $x_{n+1}=\Pi_{C_{n+1}} x_{1} \in C_{n+1} \subset C_{n}$, we have $\phi\left(x_{n+1}, y_{n}\right) \leq \phi\left(x_{n+1}\right.$, $\left.x_{n}\right)+K_{n} \rightarrow 0$ as $n \rightarrow \infty$. By Lemma 2.5 , it follows that

$$
\lim _{n \rightarrow \infty}\left\|x_{n+1}-y_{n}\right\|=0
$$

From $\lim _{n \rightarrow \infty} x_{n}=p$, we also have

$$
\lim _{n \rightarrow \infty} y_{n}=p
$$

By using the triangle inequality, we get $\left\|x_{n}-y_{n}\right\| \leq\left\|x_{n}-x_{n+1}\right\|+\left\|x_{n+1}-y_{n}\right\| \rightarrow 0$ as $n \rightarrow \infty$. Since $J$ is uniformly norm-to-norm continuous, we obtain $\left\|J x_{n}-J y_{n}\right\| \rightarrow 0$ as $n \rightarrow \infty$. On the other hand, we note that

$$
\begin{aligned}
\phi\left(q, x_{n}\right)-\phi\left(q, y_{n}\right) & =\left\|x_{n}\right\|^{2}-\left\|y_{n}\right\|^{2}-2\left\langle q, J x_{n}-J y_{n}\right\rangle \\
& \leq\left\|x_{n}-y_{n}\right\|\left(\left\|x_{n}+y_{n}\right\|\right)+2\|q\|\left\|J x_{n}-J y_{n}\right\| .
\end{aligned}
$$

In view of $\left\|x_{n}-y_{n}\right\| \rightarrow 0$ and $\left\|J x_{n}-J y_{n}\right\| \rightarrow 0$ as $n \rightarrow \infty$, we obtain that

$$
\phi\left(q, x_{n}\right)-\phi\left(q, y_{n}\right) \rightarrow 0 \quad \text { as } n \rightarrow \infty
$$


From Lemma 2.17, we have

$$
\begin{aligned}
\phi\left(q, y_{n}\right)= & \phi\left(q, J^{-1}\left[\alpha_{n} J x_{n}+\beta_{n} J w_{n}+\gamma_{n} J u_{n}\right]\right) \\
\leq & \|q\|^{2}-2\left\langle q, \alpha_{n} J x_{n}+\beta_{n} J w_{n}+\gamma_{n} J u_{n}\right\rangle+\left\|\alpha_{n} J x_{n}+\beta_{n} J w_{n}+\gamma_{n} J u_{n}\right\|^{2} \\
& -\alpha_{n} \beta_{n} g\left(\left\|J x_{n}-J w_{n}\right\|\right) \\
= & \alpha_{n} \phi\left(q, x_{n}\right)+\beta_{n} \phi\left(q, w_{n}\right)+\gamma_{n} \phi\left(q, u_{n}\right)-\alpha_{n} \beta_{n} g\left(\left\|J x_{n}-J w_{n}\right\|\right) \\
\leq & \phi\left(q, x_{n}\right)+K_{n}-\alpha_{n} \beta_{n} g\left(\left\|J x_{n}-J w_{n}\right\|\right) .
\end{aligned}
$$

It follows from $\liminf _{n \rightarrow \infty} \alpha_{n} \beta_{n}>0$, (3.16), (3.11) and the property of $g$ that

$$
\lim _{n \rightarrow \infty}\left\|J x_{n}-J w_{n}\right\|=0
$$

Since $J^{-1}$ is uniformly norm-to-norm continuous, we obtain

$$
\lim _{n \rightarrow \infty}\left\|x_{n}-w_{n}\right\|=0
$$

From (3.10) it follows that

$$
\lim _{n \rightarrow \infty}\left\|w_{n}-p\right\|=0
$$

For $w_{n} \in S^{n} x_{n}$, generate a sequence $\left\{s_{n}\right\}$ by

$$
\begin{aligned}
& s_{2} \in S w_{1} \subset S^{2} x_{1}, \\
& s_{3} \in S w_{2} \subset S^{3} x_{2}, \\
& s_{4} \in S w_{3} \subset S^{4} x_{3}, \\
& \vdots \\
& s_{n+1} \in S w_{n} \subset S^{n+1} x_{n} .
\end{aligned}
$$

On the other hand, we have $\left\|s_{n+1}-p\right\| \leq\left\|s_{n+1}-w_{n}\right\|+\left\|w_{n}-p\right\|$. Since $S$ is uniformly asymptotically regular, it follows that

$$
\lim _{n \rightarrow \infty}\left\|s_{n+1}-p\right\|=0
$$

we have

$$
\lim _{n \rightarrow \infty}\left\|S^{n+1} x_{n}-p\right\|=0
$$

that is, $S S^{n} x_{n} \rightarrow p$ as $n \rightarrow \infty$. From the closedness of $S$, we have $p \in F(S)$.

(b) We show that $p \in A^{-1} 0$.

From the definition of $C_{n+1}$ and $x_{n+1}=\Pi_{C_{n+1}} x_{1} \in C_{n+1} \subset C_{n}$, we have $\phi\left(x_{n+1}, z_{n}\right) \leq \phi\left(x_{n+1}\right.$, $\left.x_{n}\right)+K_{n} \rightarrow 0$ as $n \rightarrow \infty$. By Lemma 2.5 , it follows that $\lim _{n \rightarrow \infty}\left\|x_{n+1}-z_{n}\right\|=0$. By the 
triangle inequality, we get $\left\|x_{n}-z_{n}\right\| \leq\left\|x_{n}-x_{n+1}\right\|+\left\|x_{n+1}-z_{n}\right\| \rightarrow 0$ as $n \rightarrow \infty$. From $\lim _{n \rightarrow \infty}\left\|z_{n}-x_{n}\right\|=0$ and from (3.10), it follows that

$$
\lim _{n \rightarrow \infty} z_{n}=p
$$

Since $J$ is uniformly norm-to-norm continuous, we also have

$$
\lim _{n \rightarrow \infty}\left\|J z_{n}-J x_{n}\right\|=0 .
$$

Hence, from the definition of the sequence $\left\{z_{n}\right\}$, it follows that

$$
\left\|A x_{n}\right\|=\frac{\left\|J z_{n}-J x_{n}\right\|}{\lambda_{n}} .
$$

From (3.23) and the definition of the sequence $\left\{\lambda_{n}\right\}$, we have

$$
\lim _{n \rightarrow \infty}\left\|A x_{n}\right\|=0,
$$

that is,

$$
\lim _{n \rightarrow \infty} A x_{n}=0 .
$$

Since $A$ is Lipschitz continuous, it follows from (3.10) that

$$
A p=0 .
$$

Again, since $A$ is Lipschitz continuous and monotone so it is maximal monotone. It follows from Lemma 2.18 that $p \in A^{-1} 0$.

(c) We show that $p \in E P(f)$.

From $x_{n}, y_{n} \rightarrow 0$ and $K_{n} \rightarrow 0$ as $n \rightarrow \infty$ and applying (3.7) for any $q \in F$, we get $\lim _{n \rightarrow \infty} \phi\left(q, u_{n}\right) \rightarrow \phi(q, p)$, it follows that

$$
\begin{aligned}
\phi\left(u_{n}, x_{n}\right) & =\phi\left(T_{r_{n}}, x_{n}\right) \\
& \leq \phi\left(q, x_{n}\right)-\phi\left(q, T_{r_{n}} x_{n}\right) \\
& =\phi\left(q, x_{n}\right)-\phi\left(q, u_{n}\right) .
\end{aligned}
$$

Taking limit as $n \rightarrow \infty$ on the both sides of the inequality, we have $\lim _{n \rightarrow \infty} \phi\left(u_{n}, x_{n}\right)=0$. From Lemma 2.5 , it follows that

$$
\lim _{n \rightarrow \infty}\left\|u_{n}-x_{n}\right\|=0
$$

and

$$
\lim _{n \rightarrow \infty} u_{n}=p
$$

Since $J$ is uniformly norm-to-norm continuous on bounded subsets of $E$, we obtain

$$
\lim _{n \rightarrow \infty}\left\|J u_{n}-J z_{n}\right\|=0 .
$$


Since $r_{n}>0$ for all $n \geq 1$, we have $\frac{\left\|J u_{n}-J z_{n}\right\|}{r_{n}} \rightarrow 0$ as $n \rightarrow \infty$ and

$$
f\left(u_{n}, y\right)+\frac{1}{r_{n}}\left\langle y-u_{n}, J u_{n}-J z_{n}\right\rangle \geq 0, \quad \forall y \in C .
$$

From (A2), the fact that

$$
\begin{aligned}
\left\|y-u_{n}\right\| \frac{\left\|J u_{n}-J z_{n}\right\|}{r_{n}} & \geq \frac{1}{r_{n}}\left\langle y-u_{n}, J u_{n}-J z_{n}\right\rangle \\
& \geq-f\left(u_{n}, y\right) \\
& \geq f\left(y, u_{n}\right), \quad \forall y \in C,
\end{aligned}
$$

taking the limit as $n \rightarrow \infty$ in the above inequality and from the fact that $u_{n} \rightarrow p$ as $n \rightarrow \infty$, it follows that $f(y, p) \leq 0$ for all $y \in C$. For any $0<t<1$, define $y_{t}=t y+(1-t) p$. Then $y_{t} \in C$, which implies that $f\left(y_{t}, p\right) \leq 0$. Thus it follows from (A1) that

$$
0=f\left(y_{t}, y_{t}\right) \leq t f\left(y_{t}, y\right)+(1-t) \theta\left(y_{t}, p\right) \leq t f\left(y_{t}, y\right)
$$

and so $f\left(y_{t}, y\right) \geq 0$. From (A3) we have $f(p, y) \geq 0$ for all $y \in C$ and so $p \in E P(f)$. Hence, by (a), (b) and (c), that is, $p \in F(S) \cap A^{-1} 0 \cap E P(f)$.

Finally, we show that $p=\Pi_{F} x_{1}$. From $x_{n}=\Pi_{C_{n}} x_{1}$, we have $\left\langle J x_{1}-J x_{n}, x_{n}-z\right\rangle \geq 0$ for all $z \in C_{n}$. Since $F \subset C_{n}$, we also have

$$
\left\langle J x_{1}-J x_{n}, x_{n}-\hat{p}\right\rangle \geq 0, \quad \forall \hat{p} \in F
$$

Taking limit $n \rightarrow \infty$, we obtain

$$
\left\langle x_{1}-J p, p-\hat{p}\right\rangle \geq 0, \quad \forall \hat{p} \in F .
$$

By Lemma 2.6, we can conclude that $p=\Pi_{F} x_{1}$ and $x_{n} \rightarrow p$ as $n \rightarrow \infty$. The proof is completed.

Next, we define $z_{n}=\Pi_{C} J^{-1}\left(J x_{n}-\lambda_{n} A x_{n}\right)$ and assume that $\|A y\| \leq\|A y-A u\|$ for all $y \in C$ and $u \in V I(A, C) \neq \emptyset$. We can prove the strong convergence theorem for finding the set of solutions of the variational inequality problem in a real uniformly smooth and 2 -uniformly convex Banach space.

Remark 3.2 (Qin et al. [7]) Let $\Pi_{C}$ be the generalized projection from a smooth strictly convex and reflexive Banach space $E$ onto a nonempty closed convex subset $C$ of $E$. Then $\Pi_{C}$ is a closed quasi- $\phi$-nonexpansive mapping from $E$ onto $C$ with $F\left(\Pi_{C}\right)=C$.

Corollary 3.3 Let $C$ be a nonempty closed and convex subset of a uniformly smooth and 2-uniformly convex Banach space $E$. Let $f$ be a bifunction from $C \times C$ to $\mathbb{R}$ satisfying conditions (A1)-(A4), and let $A$ be an $\alpha$-inverse-strongly monotone mapping of $C$ into $E^{*}$ satisfying $\|A y\| \leq\|A y-A u\|$ for all $y \in C$ and $u \in V I(A, C) \neq \emptyset$. Let $S: C \rightarrow 2^{C}$ be a closed and total quasi- $\phi$-asymptotically nonexpansive multi-valued mapping with nonnegative real sequences $v_{n}, \mu_{n}$ with $v_{n} \rightarrow 0, \mu_{n} \rightarrow 0$ as $n \rightarrow \infty$ and a strictly increasing continuous 
function $\psi: \mathbb{R}^{+} \rightarrow \mathbb{R}^{+}$with $\psi(0)=0$. Assume that $S$ is uniformly asymptotically regular on $C$ with $\mu_{1}=0$ and $F:=F(S) \cap E P(f) \cap V I(A, C) \neq \emptyset$. For arbitrary $x_{1} \in C, C_{1}=C$, generate a sequence $\left\{x_{n}\right\}$ by

$$
\left\{\begin{array}{l}
z_{n}=\Pi_{C} J^{-1}\left(J x_{n}-\lambda_{n} A x_{n}\right), \\
u_{n}=T_{r_{n}} x_{n}, \\
y_{n}=J^{-1}\left(\alpha_{n} J x_{n}+\beta_{n} J w_{n}+\gamma_{n} J u_{n}\right), \quad w_{n} \in S^{n} x_{n}, \\
C_{n+1}=\left\{v \in C_{n}: \phi\left(v, y_{n}\right) \leq \phi\left(v, z_{n}\right) \leq \phi\left(v, x_{n}\right)+K_{n}\right\}, \\
x_{n+1}=\Pi_{C_{n+1}} x_{1}, \quad n \in \mathbb{N},
\end{array}\right.
$$

where $K_{n}=v_{n} \sup _{q \in F} \psi\left(\phi\left(q, x_{n}\right)\right)+\mu_{n}$. Assume that the control sequences $\left\{\alpha_{n}\right\},\left\{\beta_{n}\right\},\left\{\gamma_{n}\right\}$, $\left\{\lambda_{n}\right\}$ and $\left\{r_{n}\right\}$ satisfy the following conditions:

1. $\left\{\alpha_{n}\right\},\left\{\beta_{n}\right\}$ and $\left\{\gamma_{n}\right\}$ are sequences in $(0,1)$ such that $\alpha_{n}+\beta_{n}+\gamma_{n}=1$, $\liminf _{n \rightarrow \infty} \alpha_{n} \beta_{n}>0$,

2. $\left\{\lambda_{n}\right\} \subset[a, b]$ for some $a, b$ with $0<a<b<\frac{c^{2} \alpha}{2}$ and $\frac{1}{c}$ is the 2-uniformly convex constant of $E$,

3. $\left\{r_{n}\right\} \subset[d, \infty)$ for some $d>0$,

then $\left\{x_{n}\right\}$ converges strongly to $\Pi_{F} x_{1}$.

Proof For $q \in F$ and $\Pi_{C}$ is quasi- $\phi$-nonexpansive mapping, we have

$$
\phi\left(q, z_{n}\right)=\phi\left(q, \Pi_{C} J^{-1}\left(J x_{n}-\lambda_{n} A x_{n}\right)\right) \leq \phi\left(q, J^{-1}\left(J x_{n}-\lambda_{n} A x_{n}\right)\right) .
$$

So, we can show that $p \in V I(A, C)$.

Define $B \subset E \times E^{*}$ by Theorem 2.14, $B$ is maximal monotone and $B^{-1} 0=V I(A, C)$. Let $(z, w) \in G(B)$. Since $w \in B z=A z+N_{C}(z)$, we get $w-A z \in N_{C}(z)$.

From $z_{n} \in C$, we have

$$
\left\langle z-z_{n}, w-A z\right\rangle \geq 0 .
$$

On the other hand, since $z_{n}=\Pi_{C} J^{-1}\left(J x_{n}-\lambda_{n} A x_{n}\right)$. Then, by Lemma 2.6, we have

$$
\left\langle z-z_{n}, J z_{n}-\left(J x_{n}-\lambda_{n} A x_{n}\right)\right| \geq 0,
$$

and thus

$$
\left\langle z-z_{n}, \frac{J x_{n}-J z_{n}}{\lambda_{n}}-A x_{n}\right\rangle \leq 0
$$

It follows from (3.31) and (3.32) that

$$
\begin{aligned}
\left\langle z-z_{n}, w\right\rangle & \geq\left\langle z-z_{n}, A z\right\rangle \\
& \geq\left\langle z-z_{n}, A z\right\rangle+\left\langle z-z_{n}, \frac{J x_{n}-J z_{n}}{\lambda_{n}}-A x_{n}\right\rangle \\
& =\left\langle z-z_{n}, A z-A x_{n}\right\rangle+\left\langle z-z_{n}, \frac{J x_{n}-J z_{n}}{\lambda_{n}}\right\rangle
\end{aligned}
$$




$$
\begin{aligned}
& =\left\langle z-z_{n}, A z-A z_{n}\right\rangle+\left\langle z-z_{n}, A z_{n}-A x_{n}\right\rangle+\left\langle z-z_{n}, \frac{J x_{n}-J z_{n}}{\lambda_{n}}\right\rangle \\
& \geq-\left\|z-z_{n}\right\| \frac{\left\|z_{n}-x_{n}\right\|}{\alpha}-\left\|z-z_{n}\right\| \frac{\left\|J x_{n}-J z_{n}\right\|}{a} \\
& \geq-M\left(\frac{\left\|z_{n}-x_{n}\right\|}{\alpha}+\frac{\left\|J x_{n}-J z_{n}\right\|}{a}\right),
\end{aligned}
$$

where $M=\sup _{n \geq 1}\left\|z-z_{n}\right\|$. From $\left\|x_{n}-z_{n}\right\| \rightarrow 0$ as $n \rightarrow \infty$ and (3.23), taking $\lim _{n \rightarrow \infty}$ on the both sides of the equality above, we have $\langle z-p, w\rangle \geq 0$. By the maximality of $B$, we have $p \in B^{-1} 0$, that is, $p \in V I(A, C)$. From Theorem 3.1, we have $p \in F(S) \cap E P(f) \cap V I(A, C)$. The proof is completed.

Let $A$ be a strongly monotone mapping with constant $k$, Lipschitz with constant $L>0$, that is,

$$
\|A x-A y\| \leq L\|x-y\|, \quad \forall x, y \in D(A)
$$

which implies that

$$
\frac{1}{L}\|A x-A y\| \leq\|x-y\|, \quad \forall x, y \in D(A) .
$$

It follows that

$$
\langle A x-A y, x-y\rangle \geq k\|x-y\|^{2} \geq \frac{k}{L}\|A x-A y\|^{2}
$$

hence $A$ is $\alpha$-inverse-strongly monotone with $\alpha=\frac{k}{L}$. Therefore, we have the following corollaries.

Corollary 3.4 Let $C$ be a nonempty closed and convex subset of a uniformly smooth and 2-uniformly convex Banach space $E$. Let $f$ be a bifunction from $C \times C$ to $\mathbb{R}$ satisfying conditions (A1)-(A4), and let $A: E \rightarrow E^{*}$ be a strongly monotone mapping with constant $k$, Lipschitz with constant $L>0$. Let $S: C \rightarrow 2^{C}$ be a closed and total quasi$\phi$-asymptotically nonexpansive multi-valued mapping with nonnegative real sequences $v_{n}, \mu_{n}$ with $v_{n} \rightarrow 0, \mu_{n} \rightarrow 0$ as $n \rightarrow \infty$ and a strictly increasing continuous function $\psi: \mathbb{R}^{+} \rightarrow \mathbb{R}^{+}$with $\psi(0)=0$. Assume that $S$ is uniformly asymptotically regular on $C$ with $\mu_{1}=0$ and $F:=F(S) \cap E P(f) \cap A^{-1} 0 \neq \emptyset$. For arbitrary $x_{1} \in C, C_{1}=C$, a sequence $\left\{x_{n}\right\}$ is generated by

$$
\left\{\begin{array}{l}
z_{n}=J^{-1}\left(J x_{n}-\lambda_{n} A x_{n}\right), \\
u_{n}=T_{r_{n}} z_{n}, \\
y_{n}=J^{-1}\left(\alpha_{n} J x_{n}+\beta_{n} J w_{n}+\gamma_{n} J u_{n}\right), \quad w_{n} \in S^{n} x_{n}, \\
C_{n+1}=\left\{v \in C_{n}: \phi\left(v, y_{n}\right) \leq \phi\left(v, z_{n}\right) \leq \phi\left(v, x_{n}\right)+K_{n}\right\}, \\
x_{n+1}=\Pi_{C_{n+1}} x_{1}, \quad n \in \mathbb{N},
\end{array}\right.
$$

where $K_{n}=v_{n} \sup _{q \in F} \psi\left(\phi\left(q, x_{n}\right)\right)+\mu_{n}$. Assume that the control sequences $\left\{\alpha_{n}\right\},\left\{\beta_{n}\right\},\left\{\gamma_{n}\right\}$, $\left\{\lambda_{n}\right\}$ and $\left\{r_{n}\right\}$ satisfy the following conditions: 
1. $\left\{\alpha_{n}\right\},\left\{\beta_{n}\right\}$ and $\left\{\gamma_{n}\right\}$ are sequences in $(0,1)$ such that $\alpha_{n}+\beta_{n}+\gamma_{n}=1$, $\liminf _{n \rightarrow \infty} \alpha_{n} \beta_{n}>0$,

2. $\left\{\lambda_{n}\right\} \subset[a, b]$ for some $a, b$ with $0<a<b<\frac{c^{2} k}{2 L}$ and $\frac{1}{c}$ is the 2-uniformly convex constant of $E$,

3. $\left\{r_{n}\right\} \subset[d, \infty)$ for some $d>0$,

then $\left\{x_{n}\right\}$ converges strongly to $\Pi_{F} x_{1}$.

Corollary 3.5 Let $C$ be a nonempty closed and convex subset of a uniformly smooth and 2-uniformly convex Banach space E. Let $f$ be a bifunction from $C \times C$ to $\mathbb{R}$ satisfying conditions (A1)-(A4), and let $A: C \rightarrow E^{*}$ be a strongly monotone mapping with constant $k$, Lipschitz with constant $L>0$ satisfying $\|A y\| \leq\|A y-A u\|$ for all $y \in C$ and $u \in V I(A, C) \neq \emptyset$. Let $S: C \rightarrow 2^{C}$ be a closed and total quasi- $\phi$-asymptotically nonexpansive multi-valued mapping with nonnegative real sequences $v_{n}, \mu_{n}$ with $v_{n} \rightarrow 0, \mu_{n} \rightarrow 0$ as $n \rightarrow \infty$ and a strictly increasing continuous function $\psi: \mathbb{R}^{+} \rightarrow \mathbb{R}^{+}$with $\psi(0)=0$. Assume that $S$ is uniformly asymptotically regular on $C$ with $\mu_{1}=0$ and $F:=F(S) \cap E P(f) \cap V I(A, C) \neq \emptyset$. For arbitrary $x_{1} \in C, C_{1}=C$, generate a sequence $\left\{x_{n}\right\}$ by

$$
\left\{\begin{array}{l}
z_{n}=\Pi_{C} J^{-1}\left(J x_{n}-\lambda_{n} A x_{n}\right), \\
u_{n}=T_{r_{n}} x_{n}, \\
y_{n}=J^{-1}\left(\alpha_{n} J x_{n}+\beta_{n} J w_{n}+\gamma_{n} J u_{n}\right), \quad w_{n} \in S^{n} x_{n}, \\
C_{n+1}=\left\{v \in C_{n}: \phi\left(v, y_{n}\right) \leq \phi\left(v, z_{n}\right) \leq \phi\left(v, x_{n}\right)+K_{n}\right\}, \\
x_{n+1}=\Pi_{C_{n+1}} x_{1}, \quad n \in \mathbb{N},
\end{array}\right.
$$

where $K_{n}=v_{n} \sup _{q \in F} \psi\left(\phi\left(q, x_{n}\right)\right)+\mu_{n}$. Assume that the control sequences $\left\{\alpha_{n}\right\},\left\{\beta_{n}\right\},\left\{\gamma_{n}\right\}$, $\left\{\lambda_{n}\right\}$ and $\left\{r_{n}\right\}$ satisfy the following conditions:

1. $\left\{\alpha_{n}\right\},\left\{\beta_{n}\right\}$ and $\left\{\gamma_{n}\right\}$ are sequences in $(0,1)$ such that $\alpha_{n}+\beta_{n}+\gamma_{n}=1$, $\liminf _{n \rightarrow \infty} \alpha_{n} \beta_{n}>0$,

2. $\left\{\lambda_{n}\right\} \subset[a, b]$ for some $a, b$ with $0<a<b<\frac{c^{2} k}{2 L}$ and $\frac{1}{c}$ is the 2-uniformly convex constant of $E$,

3. $\left\{r_{n}\right\} \subset[d, \infty)$ for some $d>0$, then $\left\{x_{n}\right\}$ converges strongly to $\Pi_{F} x_{1}$.

Let $F$ be a Fréchet differentiable functional in a Banach space $E$ and $\nabla F$ be the gradient of $F$, denote $(\nabla F)^{-1} 0=\left\{x \in E: F(x)=\min _{y \in E} F(y)\right\}$. Baillon and Haddad [34] proved the following lemma.

Lemma 3.6 (Baillon and Haddad [34]) Let E be a Banach space. Let F be a continuously Fréchet differentiable convex functional on $E$ and $\nabla F$ be the gradient of $F$. If $\nabla F$ is $\frac{1}{\alpha}$ Lipschitz continuous, then $\nabla F$ is an $\alpha$-inverse strongly monotone mapping.

We replace $A$ in Theorem 3.1 by $\nabla F$, then we can obtain the following corollary.

Corollary 3.7 Let $C$ be a nonempty closed and convex subset of a uniformly smooth and 2-uniformly convex Banach space E. Let $f$ be a bifunction from $C \times C$ to $\mathbb{R}$ satisfying conditions (A1)-(A4). Let $F$ be a continuously Fréchet differentiable convex functional 
on $E$ and $\nabla F$ be $\frac{1}{\alpha}$-Lipschitz continuous. Let $S: C \rightarrow 2^{C}$ be a closed and total quasi$\phi$-asymptotically nonexpansive multi-valued mapping with nonnegative real sequences $v_{n}, \mu_{n}$ with $v_{n} \rightarrow 0, \mu_{n} \rightarrow 0$ as $n \rightarrow \infty$ and a strictly increasing continuous function $\psi: \mathbb{R}^{+} \rightarrow \mathbb{R}^{+}$with $\psi(0)=0$. Assume that $S$ is uniformly asymptotically regular on $C$ with $\mu_{1}=0$ and $F:=F(S) \cap F(T) \cap E P(f) \cap A^{-1} 0 \neq \emptyset$. For an initial point $x_{1} \in E, C_{1}=C$, define the sequence $\left\{x_{n}\right\}$ by

$$
\left\{\begin{array}{l}
z_{n}=J^{-1}\left(J x_{n}-\lambda_{n} \nabla F x_{n}\right), \\
u_{n}=T_{r_{n}} x_{n}, \\
y_{n}=J^{-1}\left(\alpha_{n} J x_{n}+\beta_{n} J w_{n}+\gamma_{n} J u_{n}\right), \quad w_{n} \in S^{n} x_{n}, \\
C_{n+1}=\left\{v \in C_{n}: \phi\left(v, y_{n}\right) \leq \phi\left(v, z_{n}\right) \leq \phi\left(v, x_{n}\right)+K_{n}\right\}, \\
x_{n+1}=\Pi_{C_{n+1}} x_{1}, \quad n \in \mathbb{N},
\end{array}\right.
$$

where $\mu_{n}=\sup \left\{\mu_{n}^{S}, \mu_{n}^{T}\right\}, v_{n}=\sup \left\{v_{n}^{S}, v_{n}^{T}\right\}, \psi=\sup \left\{\psi^{S}, \psi^{T}\right\}, k_{n}=v_{n} \sup _{q \in \mathcal{F}} \psi\left(\phi\left(q, x_{n}\right)\right)+\mu_{n}$. Assume that the control sequences $\left\{\alpha_{n}\right\},\left\{\beta_{n}\right\},\left\{\gamma_{n}\right\},\left\{\lambda_{n}\right\}$ and $\left\{r_{n}\right\}$ satisfy the following conditions:

1. $\left\{\alpha_{n}\right\},\left\{\beta_{n}\right\}$ and $\left\{\gamma_{n}\right\}$ are sequences in $(0,1)$ such that $\alpha_{n}+\beta_{n}+\gamma_{n}=1$, $\liminf _{n \rightarrow \infty} \alpha_{n} \beta_{n}>0$ and $\liminf _{n \rightarrow \infty} \alpha_{n} \gamma_{n}>0$,

2. $\left\{\lambda_{n}\right\} \subset[a, b]$ for some $a, b$ with $0<a<b<\frac{c^{2} \alpha}{2}$ and the 2 -uniformly convex constant $\frac{1}{c}$ of $E$,

3. $\left\{r_{n}\right\} \subset[d, \infty)$ for some $d>0$,

then $\left\{x_{n}\right\}$ converges strongly to $\Pi_{F} x_{1}$.

\section{Competing interests}

The author declares that they have no competing interests.

\section{Acknowledgements}

This work was supported by Thaksin University Research Fund. Moreover, the author also would like to thank Faculty of Science, Thaksin University.

Received: 11 July 2013 Accepted: 9 October 2013 Published: 11 Nov 2013

\section{References}

1. Alber, YI: Metric and generalized projection operators in Banach spaces: properties and applications. In: Kartsatos, A (ed.) Theory and Applications of Nonlinear Operators of Accretive and Monotone Type, pp. 15-50. Dekker, New York (1996)

2. Iiduka, $\mathrm{H}$, Takahashi, $\mathrm{W}$ : Weak convergence of a projection algorithm for variational inequalities in a Banach space. J. Math. Anal. Appl. 339, 668-679 (2008)

3. liduka, $\mathrm{H}$, Takahashi, W: Strong convergence studied by a hybrid type method for monotone operators in a Banach space. Nonlinear Anal. 68, 3679-3688 (2008)

4. Ceng, LC, Latif, A, Yao, JC: On solutions of system of variational inequalities and fixed point problems in Banach spaces. Fixed Point Theory Appl. 2013, 176 (2013). doi:10.1186/1687-1812-2013-176

5. Kangtunyakarn, A: A new mapping for finding a common element of the sets of fixed points of two finite families of nonexpansive and strictly pseudo-contractive mappings and two sets of variational inequalities in uniformly convex and 2-smooth Banach spaces. Fixed Point Theory Appl. 2013, 157 (2013). doi:1 0.1186/1687-1812-2013-157

6. Kassay, G, Reich, S, Sabach, S: Iterative methods for solving systems of variational inequalities in reflexive Banach spaces. SIAM J. Optim. 21, 1319-1344 (2011)

7. Qin, X, Cho, YJ, Kang, SM: Convergence theorems of common elements for equilibrium problems and fixed point problems in Banach spaces. J. Comput. Appl. Math. 225, 20-30 (2009)

8. Zhou, H, Gao, G, Tan, B: Convergence theorems of a modified hybrid algorithm for a family of quasi- $\phi$-asymptotically nonexpansive mappings. J. Appl. Math. Comput. 32(2), 453-464 (2010)

9. Chang, S-S, Wang, L, Tang, Y-K, Zhao, Y-H, Ma, Z-L: Strong convergence theorems of nonlinear operator equations for countable family of multi-valued total quasi- $\phi$-asymptotically nonexpansive mappings with applications. Fixed Point Theory Appl. 2012, 69 (2012). doi:10.1186/1687-1812-2012-69

10. Takahashi, W, Zembayashi, K: Strong convergence theorem by a new hybrid method for equilibrium problems and relatively nonexpansive mappings. Fixed Point Theory Appl. 2008, Article ID 528476 (2008) 
11. Takahashi, W, Zembayashi, K: Strong and weak convergence theorems for equilibrium problems and relatively nonexpansive mappings in Banach spaces. Nonlinear Anal. 70, 45-57 (2009)

12. Cholamjiak, P: Strong convergence of projection algorithms for a family of relatively quasi-nonexpansive mappings and an equilibrium problem in Banach spaces. Optimization 60(4), 495-507 (2011)

13. Combettes, PL, Hirstoaga, SA: Equilibrium programming in Hilbert spaces. J. Nonlinear Convex Anal. 6, 117-136 (2005)

14. Iusema, AN, Sosa, W: Iterative algorithms for equilibrium problems. Optimization 52(3), 301-316 (2003)

15. Saewan, S, Kumam, P, Wattanawitoon, K: Convergence theorem based on a new hybrid projection method for finding a common solution of generalized equilibrium and variational inequality problems in Banach spaces. Abstr. Appl. Anal. 2010, Article ID 734126 (2010)

16. Ceng, LC, Al-Homidan, S, Ansari, QH, Yao, J-C: An iterative scheme for equilibrium problems and fixed points problems of strict pseudo-contraction mappings. J. Comput. Appl. Math. 223(2), 967-974 (2009)

17. Zeng, L-C, Ansari, QH, Shyu, DS, Yao, J-C: Strong and weak convergence theorems for common solutions of generalized equilibrium problems and zeros of maximal monotone operators. Fixed Point Theory Appl. 2010, Article ID 590278 (2010)

18. Ceng, LC, Ansari, QH, Yao, JC: Strong and weak convergence theorems for asymptotically strict pseudocontractive mappings in intermediate sense. J. Nonlinear Convex Anal. 11(2), 283-308 (2010)

19. Ceng, L-C, Ansari, QH, Yao, J-C: Hybrid proximal-type and hybrid shrinking projection algorithms for equilibrium problems, maximal monotone operators and relatively nonexpansive mappings. Numer. Funct. Anal. Optim. 31(7), 763-797 (2010)

20. Zeng, LC, Al-Homidan, S, Ansari, QH: Hybrid proximal-type algorithms for generalized equilibrium problems, maximal monotone operators and relatively nonexpansive mappings. Fixed Point Theory Appl. 2011, Article ID 973028 (2011)

21. Zeng, L-C, Ansari, QH, Schaible, S, Yao, J-C: Hybrid viscosity approximate method for zeros of $m$-accretive operators in Banach spaces. Numer. Funct. Anal. Optim. 33(2), 142-165 (2012)

22. Cioranescu, I: Geometry of Banach Spaces, Duality Mappings and Nonlinear Problems. Kluwer Academic, Dordrecht (1990)

23. Reich, S: Geometry of Banach spaces, duality mappings and nonlinear problems. Bull. Am. Math. Soc. 26, 367-370 (1992)

24. Beauzamy, B: Introduction to Banach Spaces and Their Geometry, 2nd edn. North-Holland, Amsterdam (1985)

25. Xu, HK: Inequalities in Banach spaces with applications. Nonlinear Anal. 16, 1127-1138 (1991)

26. Zalinescu, C: On uniformly convex functions. J. Math. Anal. Appl. 95, 344-374 (1983)

27. Kamimura, S, Takahashi, W: Strong convergence of a proximal-type algorithm in a Banach space. SIAM J. Optim. 13 938-945 (2002)

28. Blum, E, Oettli, W: From optimization and variational inequalities to equilibrium problems. Math. Stud. 63, 123-145 (1994)

29. Kohsaka, F, Takahashi, W: Existence and approximation of fixed points of firmly nonexpansive-type mappings in Banach spaced. SIAM J. Optim. 19(2), 824-835 (2008)

30. Rockafellar, RT: On the maximality of sums of nonlinear monotone operators. Trans. Am. Math. Soc. 149, 75-88 (1970)

31. Takahashi, W: Nonlinear Functional Analysis. Yokohama Publishers, Yokohama (2000)

32. Cho, YJ, Zhou, HY, Guo, G: Weak and strong convergence theorems for three-step iterations with errors for asymptotically nonexpansive mappings. Comput. Math. Appl. 47, 707-717 (2004)

33. Pascali, D, Sburlan, S: Nonlinear Mappings of Monotone Type. Editura Academiae Bucaresti, Romania (1978)

34. Baillon, JB, Haddad, G: Quelques propriétés des opérateurs andle-bornés et $n$-cycliquement monotones. Isr. J. Math 26, 137-150 (1977)

10.1186/1687-1812-2013-297

Cite this article as: Saewan: Strong convergence theorem for total quasi- $\phi$-asymptotically nonexpansive mappings in a Banach space. Fixed Point Theory and Applications 2013, 2013:297

\section{Submit your manuscript to a SpringerOpen ${ }^{\circ}$ journal and benefit from:}

- Convenient online submission

Rigorous peer review

- Immediate publication on acceptance

- Open access: articles freely available online

- High visibility within the field

- Retaining the copyright to your article 